\title{
Adhesion Molecules on Normotensive and Hypertensive Rat Brain Endothelial Cells (43706)
}

\author{
R. M. McCarron, ${ }^{*, 1}$ L. Wang, ${ }^{*}$ A.-L. Siren, $\dagger$ M. Spatz, ${ }^{*}$ and J. M. Hallenbeck* \\ Stroke Branch, National Institute of Neurological Disorders and Stroke, National Institutes of Health, Bethesda, \\ Maryland 20892 and Department of Neurology, $\dagger$ Uniformed Services University of the Health Sciences, \\ Bethesda, Maryland 20814
}

\begin{abstract}
The intercellular adhesion of circulating leukocytes to vascular endothellum is a prerequisite for leukocyte emigration from the blood to extravascular tissues. This process is facilltated by adhesion molecules on the surfaces of both the vascular endothelial cells and the leukocytes. The experiments presented here demonstrate for the first time that the leukocyte adhesion receptor, intercellular adhesion molecule-1, is constitutively expressed on cultured cerebromicrovascular endothelial cell lines derived from both spontaneously hypertensive (SHR) rats and normotensive WistarKyoto (WKY) rats. Both cultures contained similar numbers of cells constitutively expressing this adhesion molecule (31.4\% and $29.6 \%$, respectively). Adhesion molecule expression was up-regulated by interleukin-1ß, tumor necrosis factor- $\alpha$, Interferon- $\gamma$ and lipopolysaccharide in a dose- and time-dependent manner. Both cultures exhiblted similar maximum levels of adhesion molecule up-regulation to optimal concentrations of all three cytokines. However, SHR endothelial cells were more sensitive to all three cytokines; significantly higher levels of intercellular adhesion molecule-1 expression were seen on SHR as opposed to WKY endothellal cells cultured with sub-optimal cytokine concentrations. It was also observed that lipopolysaccharide up-regulated intercellular adhesion molecule-1 expression on SHR endothellal cells to a greater extent than on WKY endothelial cells.

The findings that intercellular adhesion molecule-1 can be up-regulated to a greater degree on SHR endothellal cells may have important implications for In vivo perivascular leukocyte accumulation under hypertensive conditions. These observations indicate a possible mechanism by which hypertension may predlspose to the development of disorders such as atherosclerosis and stroke. [P.S.E.B.M. 1994, Vol 205]
\end{abstract}

$\mathrm{T}$ The adhesion of leukocytes to vascular endothelial cells $(E C)$ is an initial, critical step required for their emigration from the blood stream to extravascular tissues (1-3). The adhesion event is facilitated by a variety of adhesion molecules present on the surfaces of EC (e.g., intercellular adhesion molecule-1 [ICAM-1], vascular cell adhesion molecule-1,

\footnotetext{
1 To whom requests for reprints should be addressed at Bldg. 36, Room 4D04, Stroke Branch, NINDS, National Institutes of Health, 9000 Rockville Pike, Bethesda, MD 20892.
}

Received April 5, 1993. [P.S.E.B.M. 1994, Vol 205]

Accepted November 23, 1993.

Copyright $(\mathcal{O} 1994$ by the Society for Experimental Biology and Medicine etc.) which interact with their complementary ligands (membrane-associated glycoproteins [integrins]) present on leukocytes (e.g., LFA-1, VLA-4, etc.). Although essential for host repair and defense, intercellular adhesion processes may also result in endothelial injury which occurs during allograft rejection (4) and in chronic inflammatory disorders such as autoimmune murine lupus nephritis (5) and atherosclerosis (6). The expression of adhesion molecules by cerebromicrovascular EC, as well as the resultant adherence of leukocytes and their migration have also been studied in disease states, including hemorrhagic shock, cerebral ischemic stroke, atherosclerosis, and multiple sclerosis (7-12). These disorders are characterized, in part, by leukocyte adhesion to cerebromicrovascular $\mathrm{EC}$ and subsequent extravascular migration. The above studies have documented the expression of adhesion molecules on cerebromicrovascular EC and the 
resultant interactions with various circulating leukocytes.

In recent years, ICAM-1 (CD54) expression, in particular, has been demonstrated to promote adhesion of a variety of blood leukocytes including lymphocytes, neutrophils, and monocytes $(1,13-16)$. ICAM-1 is very strongly expressed by certain nonhematopoietic cells, such as vascular EC, and weakly expressed by some blood mononuclear cells, such as monocytes and lymphocytes (17). ICAM-1 expression can be induced and/or up-regulated in vitro by a variety of cytokines such as interleukin- $1 \beta$ (IL-1 $\beta$ ), tumor necrosis factor- $\alpha(\mathrm{TNF} \alpha)$, and interferon- $\gamma(\mathrm{IFN} \gamma)(2$, $13,16,18)$. In addition, lipopolysaccharide (LPS), a prototypic stimulus to activate the cytokine system, also enhances ICAM expression (19).

In rats, in vitro ICAM-1 expression has been reported in EC isolated from heart (20) and peripheral lymph nodes $(21,22)$. In the present study, cerebromicrovascular endothelial cell lines derived from spontaneously hypertensive rats (SHR) and Wistar-Kyoto rats (WKY) were examined for their ability to constitutively express ICAM-1. In addition, data are also presented demonstrating the regulation of ICAM-1 expression on SHR and WKY cerebromicrovascular EC by various cytokines and LPS. The comparison of ICAM-1 expression and regulation on both SHR and WKY cerebromicrovascular EC offers a unique opportunity to examine the possible influence of hypertension, a known stroke risk factor, on EC responsiveness to proinflammatory factors.

\section{Materials and Methods}

Rats. Female Wistar-Kyoto (WKY) and spontaneously hypertensive (SHR) rats obtained from Taconic Farms (Germantown, NY) at 9 to 14 weeks of age and Sprague Dawley (SD) rats obtained from Harlan Sprague Dawley, Inc. (Indianapolis, IN) were utilized to isolate cerebromicrovascular EC used in this study. All animal procedures were in accordance with guidelines put forth in the NIH Guide for the Care and Use of Laboratory Animals.

Cerebromicrovascular EC Cultures. The isolation and cultivation of cerebromicrovascular EC from SHR, WKY and SD rats was performed with some modification as previously described (23). Propagated EC $\left(1 \times 10^{4} / 100 \mu \mathrm{l} /\right.$ well $)$ were passaged and grown to confluency on $1 \%$ gelatin-coated coverslips or 96 -well flat bottom microtiter plates at $37^{\circ} \mathrm{C}$ in humidified atmosphere of $5 \% \mathrm{CO}_{2}$ /air in $\mathrm{M}-199$ medium (containing $25 \mathrm{~m} M$ Hepes buffer, Earle's salts and L-glutamine) with $20 \%$ heat-inactivated FCS, $90 \mu \mathrm{g} / \mathrm{ml}$ heparin, 100 $\mu \mathrm{g} / \mathrm{ml}$ streptomycin, $100 \mathrm{U} / \mathrm{ml}$ penicillin $\mathrm{G}$, and 0.25 $\mu \mathrm{g} / \mathrm{ml}$ amphotericin B as fungizone (all from GIBCO, Long Island, NY) and $20 \mu \mathrm{g} / \mathrm{ml}$ endothelial cell growth supplement (Collaborative Research, Bedford, MA).
EC were positively identified by both immunocytochemistry and fluorescence-activated cell sorter (FACS) analysis using EC-specific anti-Factor VIIIrelated antigen (Accurate Chemical and Scientific Corp., Westbury, NY) as previously described (24). All cultures used in experiments described here were obtained from three separate lines and were used at 3 rd-11th passage. Confluent EC cultures derived from SHR, WKY and SD cerebral microvessels routinely exhibited characteristic cobblestone appearances, and $>95 \%$ of cells in both of these cultures stained positively for FVIII-related antigen. Prior to use in adhesion and ICAM-1 assays, cells were washed twice with Hank's Balanced Salt Solution (HBSS) followed by incubation in media alone or with indicated concentrations of recombinant IFN $\gamma$ (Genzyme Corp., Boston, MA), human IL-1 $\beta$ (Endogen, Boston, MA), recombinant human TNF $\alpha$ (Endogen), or LPS (Sigma, St. Louis, MO). All treatments were performed in quadruplicate wells. All reagents contained $<10 \mathrm{pg} / \mathrm{ml}$ endotoxin as determined by E-toxate assay kit (Sigma).

ICAM-1 Expression. EC cultures grown on gelatin-coated coverslips were extensively washed, prefixed in 1:1 mixture glacial acetic acid and methanol $\left(10 \mathrm{~min}, 4^{\circ} \mathrm{C}\right)$ and incubated at $37^{\circ} \mathrm{C}$ with $1: 50$ mouse monoclonal antibody $\left(\operatorname{IgG}_{1}\right)$ to rat ICAM-1 (clone IA29; Seikagaku Corp., Rockville, MD) for $45 \mathrm{~min}$. After incubation with 1:100 rhodamine-conjugated $\mathrm{F}\left(\mathrm{ab}^{\prime}\right)_{2}$ goat anti-mouse IgG (Accurate) for $8 \mathrm{hr}$, SHR, WKY, and SD EC cultures were observed to constitutively express ICAM-1 by indirect immunofluorescence microscopy.

The relative level of ICAM-1 expression by EC cultures was quantitated by ELISA essentially as previously described (25). Briefly, EC $\left(2 \times 10^{4}\right.$ cells $/ 50$ $\mu \mathrm{l} /$ well) cultures in 96 -well microtiter plates were incubated in the absence or presence of various concentrations of IFN $\gamma$, IL-1 $\beta, \mathrm{TNF} \alpha$, or LPS for indicated time periods at $37^{\circ} \mathrm{C}$ in $5 \% \mathrm{CO}_{2}$. Nonspecific binding sites were blocked by addition of heat-aggregated rabbit IgG (Sigma) and EC cultures were sequentially incubated at $37^{\circ} \mathrm{C}$ for $1 \mathrm{hr}$ in the presence of IA29 (1:1000); biotinylated anti-mouse IgG (1:1000); and $0.33 \mu \mathrm{g} / \mathrm{ml}$ avidin-horseradish peroxidase. All substances were obtained from Sigma and were added at $100 \mu \mathrm{l} /$ well. Plates were washed three times with PBS containing $2 \%$ BSA between each of the above steps. The reaction was developed by adding phosphatecitrate buffer $/ 0.004 \%$ O-phenylenediamine, $0.012 \%$ $\mathrm{H}_{2} \mathrm{O}_{2}\left(25^{\circ} \mathrm{C}, 30 \mathrm{~min}\right)$ and stopped by adding $4 \mathrm{~N} \mathrm{HCl}$. The optical density (O.D.) was immediately read at $490 \mathrm{~nm}$ (reference $630 \mathrm{~nm}$ ). Background values for each culture condition, obtained by incubation of EC with an irrelevant mouse antibody (RT1.B) specific for rat Class II MHC antigen (MRC OX6; Accurate) in lieu of IA29, were subtracted from test values. 
FACS Analysis. Analysis of cell populations by flow cytometry was performed on a FACS IV analyzer (Becton Dickinson FACS System, Mountain View, CA). Cerebromicrovascular cells from SHR and WKY SD rats were stained with anti-ICAM-1 or RT1.B followed by rhodamine-conjugated goat $\mathrm{F}\left(\mathrm{ab}^{\prime}\right)_{2}$ antimouse IgG (Accurate). The data are displayed as a histogram with the $y$ axis representing cell number and the $x$ axis representing fluorescence intensity (in channel units). The mean fluorescence intensity (MFI) represents semiquantitative data on the mean number of fluorescent reagent-binding molecules expressed by the cells.

\section{Results}

Confluent EC cultures derived from SHR, WKY, and SD cerebral microvessels routinely exhibited characteristic cobblestone appearances, stained positively for FVIII-related antigen and ICAM-1. The expression of ICAM-1 by SHR and WKY cerebromicrovascular EC cultures was examined by FACS analysis. A similar percentage of cells in both EC cultures constitutively expressed ICAM-1 (Table I). The mean fluorescence intensity (MFI), which is a measure of the relative level of ICAM-1 expression per cell, was also similar for both SHR (17.5 channel units) and WKY (19.1 channel units) EC. Treatment for three days with TNF $\alpha$ (1-200 U/ml) up-regulated ICAM-1 expression on both SHR and WKY EC cultures. Increases in both the percentage of cells expressing ICAM-1 and in the MFI values were correlated with the concentration of $T N F \alpha$. No significant differences in the maximum responses to TNF $\alpha(200 \mathrm{U} / \mathrm{ml})$ were seen between SHR and WKY EC cultures. However, incubation of SHR EC with $1 \mathrm{U} / \mathrm{ml} \mathrm{TNF} \alpha$ significantly increased the percentage of cells expressing ICAM-1, whereas only minor increases in the number of WKY EC expressing ICAM-1 were seen under these culture conditions. At all intermediate concentrations of TNF $\alpha$, the responsiveness of SHR EC (as assessed by

Table I. Effect of TNF $\alpha$ on ICAM-1 Expression by SHR and WKY Cerebromicrovascular EC

\begin{tabular}{rcccc}
\hline TNF $^{a}$ & \multicolumn{2}{c}{ SHR $^{b}$} & \multicolumn{2}{c}{ WKY } \\
\hline$U / m I$ & $\%$ & $M F I$ & $\%$ & $M F I$ \\
0 & 31.4 & 17.5 & 29.6 & 19.1 \\
1 & 41.8 & 21.8 & 31.3 & 18.2 \\
10 & 49.6 & 32.6 & 39.8 & 23.1 \\
100 & 61.2 & 46.9 & 59.9 & 33.4 \\
200 & 63.8 & 48.3 & 64.2 & 45.4 \\
\hline
\end{tabular}

${ }^{a}$ EC cultures were incubated in the presence of indicated concentrations TNF $\alpha$ for three days prior to analysis.

${ }^{b}$ Cerebromicrovascular EC were analyzed by FACS as described in Materials and Methods. Data indicate the (\%) percent of ICAM-1 positive cells and (MFI) mean fluorescence intensity (in channel units) of EC cultures. both the percentage of positive cells and MFI values) were greater than WKY EC. No staining was observed with an irrelevant antibody (MRC OX6) specific for rat Class II antigens.

The overall level of ICAM-1 expression by EC cultures was quantitated by ELISA. The capacity of TNF $\alpha$ to up-regulate ICAM-1 expression on both SHR and WKY EC was dose dependent (Fig. 1). Treatment with low concentrations of TNF $\alpha$ (i.e., $1-50 \mathrm{U} / \mathrm{ml}$ ) enhanced ICAM-1 expression on SHR EC to a significantly greater extent $(P<0.01)$ than on WKY EC. No significant differences in the level of ICAM-1 expression were observed between SHR and WKY EC incubated in the presence of 100 or $200 \mathrm{U} / \mathrm{ml}$ of TNF $\alpha$.

Experiments performed on other cerebrovascular EC cell lines derived from SHR, WKY and also from SD rats resulted in similar findings to those shown in Figure 1 and also demonstrate "normal" responsiveness of SD EC. In addition, other proinflammatory cytokines such as IFN $\gamma$ and IL-1 $\beta$ also increased ICAM-1 expression on these cells (Table II). Although there were differences in the overall responsiveness of EC cultures to these cytokines with TNF $\alpha>$ IL-1 $\beta>$ IFN $\gamma$, all cytokines dose-dependently up-regulated ICAM-1 expression on SHR, WKY, and SD EC cultures. In addition, incubation with suboptimal concentrations of TNF $\alpha$, IL- $1 \beta$, and IFN $\gamma$ up-regulated ICAM-1 expression on SHR EC to a greater extent

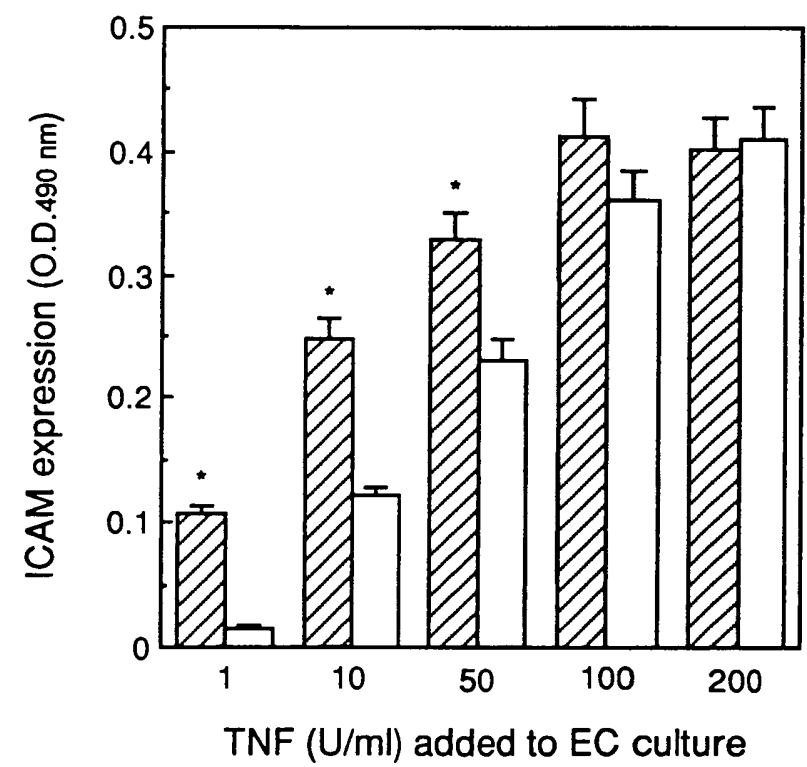

Figure 1. Effect of TNFa on ICAM-1 expression by SHR and WKY cerebromicrovascular endothelial cells (EC). Cerebrovascular EC lines isolated from SHR $(\square)$ and WKY $(\square)$ rat brain microvessels were cultured in microtiter plates for $24 \mathrm{hr}$ in the absence or presence of indicated concentrations of TNF $\alpha$. EC cultures were examined for ICAM-1 expression by ELISA as described in Materials and Methods. The values presented indicate O.D. \pm S.D. values above those obtained in EC cultures incubated in media alone $(0.123 \pm 0.008$ and $0.199 \pm 0.020$ for SHR and WKY EC, respectively); "significant difference $(P<0.01$; Student's $t$ test) between EC cultures. 
Table II. Effects of TNF $\alpha$, IFN $\gamma$, and IL-1 $\beta$ on Up-Regulation of ICAM-1 Expression on SHR, WKY, and SD Cerebrovascular EC

\begin{tabular}{|c|c|c|c|c|}
\hline & \multirow{2}{*}{$\begin{array}{l}\text { Cytokine }^{b} \\
(U / \mathrm{mI})\end{array}$} & \multicolumn{3}{|c|}{ ICAM-1 expression ${ }^{a}$} \\
\hline & & SHR EC & WKY EC & SD EC \\
\hline TNF $\alpha$ & $\begin{array}{r}1 \\
10 \\
50 \\
100\end{array}$ & $\begin{array}{l}0.146 \pm 0.003 \\
0.321 \pm 0.008 \\
0.489 \pm 0.013 \\
0.533 \pm 0.013\end{array}$ & $\begin{array}{l}0.031 \pm 0.004^{d} \\
0.181 \pm 0.007^{d} \\
0.363 \pm 0.018^{c} \\
0.479 \pm 0.031\end{array}$ & $\begin{array}{c}0.018 \pm 0.002^{d} \\
0.149 \pm 0.008^{d} \\
N D^{\theta} \\
0.426 \pm 0.032^{c}\end{array}$ \\
\hline IFNy & $\begin{array}{r}1 \\
10 \\
50 \\
100\end{array}$ & $\begin{array}{l}0.084 \pm 0.008 \\
0.169 \pm 0.009 \\
0.289 \pm 0.012 \\
0.404 \pm 0.021\end{array}$ & $\begin{array}{l}0.014 \pm 0.003^{d} \\
0.088 \pm 0.011^{c} \\
0.211 \pm 0.009^{c} \\
0.365 \pm 0.024\end{array}$ & $\begin{array}{c}0.008 \pm 0.002^{d} \\
0.068 \pm 0.010^{d} \\
\text { ND } \\
0.323 \pm 0.021^{c}\end{array}$ \\
\hline IL-1 $\beta$ & $\begin{array}{r}1 \\
5 \\
20 \\
50\end{array}$ & $\begin{array}{l}0.120 \pm 0.007 \\
0.308 \pm 0.020 \\
0.423 \pm 0.008 \\
0.494 \pm 0.032\end{array}$ & $\begin{array}{l}0.020 \pm 0.002^{d} \\
0.151 \pm 0.018^{d} \\
0.305 \pm 0.010^{c} \\
0.456 \pm 0.028\end{array}$ & $\begin{array}{c}0.021 \pm 0.003^{d} \\
0.150 \pm 0.016^{d} \\
\text { ND } \\
0.421 \pm 0.042\end{array}$ \\
\hline
\end{tabular}

a Data are presented as O.D. $490 \mathrm{~nm}$ above background \pm S.D. obtained from ELISA experiments performed as described in the Materials and Methods. Background values for SHR, WKY, and SD EC were $0.238 \pm 0.029,0.391 \pm 0.024$, and $0.316 \pm 0.025$, respectively.

${ }^{b}$ Cerebrovascular EC cultures were incubated for three days in the presence of indicated concentrations of TNF $\alpha$, IFN $\gamma$, or IL- $\beta$. c Significant difference $(P<0.01$; Student's $t$ test) compared with SHR EC.

${ }^{d}$ Significant difference $(P<0.001$; Student's $t$ test) compared with SHR EC.

ND = not done.

than on either WKY or SD EC; no significant differences in ICAM-1 expression were observed between WKY and SD EC.

The length of time required for ICAM-1 upregulation by TNF $\alpha$, IL-1 $\beta$, and IFN $\gamma$ treatment was examined. Treatment with IL-1 $\beta$ increased ICAM-1 expression as early as $4 \mathrm{hr}$ after addition to culture and reached a maximum after 16-24-hr culture (Fig. 2). A slower effect on ICAM-1 expression was observed using TNF $\alpha$ and IFN $\gamma$; very little increases in ICAM-1 expression were seen at $4 \mathrm{hr}$, but these cells also exhibited maximum levels of ICAM-1 expression after 24-hr culture. Culturing these cells with cytokines for greater than $24 \mathrm{hr}$ did not significantly increase ICAM-1 expression. No differences were observed between SHR and WKY EC.

The ability of LPS to affect ICAM-1 expression was also examined. LPS dose-dependently enhanced ICAM-1 expression on SHR, WKY, and SD EC (Fig. 3). The responsiveness of SHR EC to LPS-induced up-regulation of ICAM-1 was greater than WKY or ED EC. This enhanced responsiveness of SHR EC to LPS was observed at all LPS concentrations (0.1-100 $\mu \mathrm{g} / \mathrm{ml})$. Unlike the results obtained with cytokines, SHR EC responded to a significantly greater degree than either WKY or SD EC to optimal LPS concentrations (i.e., $100 \mu \mathrm{g} / \mathrm{ml}$ ) in three of four experiments. Incubation of SHR, WKY, and SD EC cultures with $>100 \mu \mathrm{g} / \mathrm{ml}$ LPS did not significantly increase ICAM-1 expression. In fact, incubation with LPS concentrations of $300 \mu \mathrm{g} / \mathrm{ml}$ or higher resulted in lower levels of

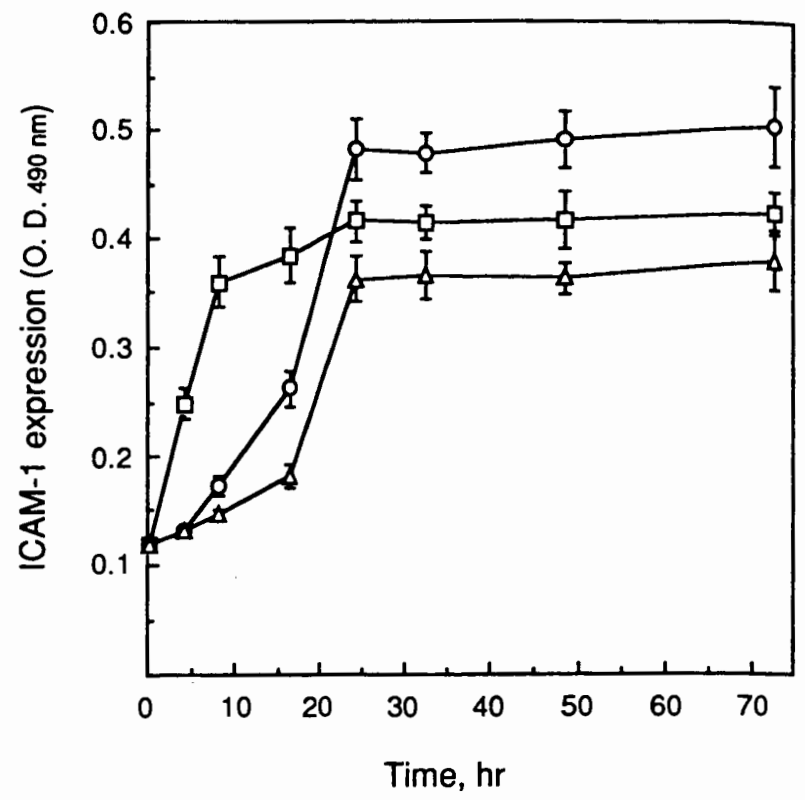

Figure 2. Time course of the effect of TNF $\alpha, I L-1 \beta$, and IFN $\gamma$ on ICAM-1 expression by cerebromicrovascular endothelial cells (EC). Cerebrovascular EC lines isolated from WKY rat brain microvessels were cultured in microtiter plates in the absence or presence of $100 \mathrm{U} / \mathrm{ml}$ IFN $\gamma(\Delta), 100 \mathrm{U} / \mathrm{ml} \mathrm{TNF} \alpha(O)$ or $50 \mathrm{U} / \mathrm{ml}$ IL-1 $\beta(\square)$ for indicated periods of time. EC cultures were examined for ICAM-1 expression by ELISA as described in Materials and Methods. The values presented indicate O.D. \pm S.D. values above those obtained in EC cultures incubated in media alone which ranged between $0.102 \pm 0.009$ and $0.215 \pm 0.020$ for all the different time periods.

ICAM-1 expression which were related to decreases in the percent of viable EC (determined by trypan blue exclusion) cultured under these conditions.

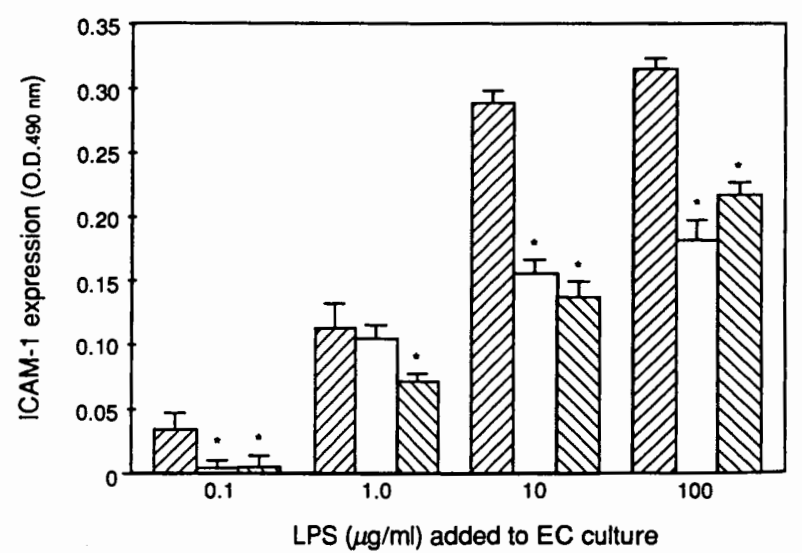

Figure 3. Effect of LPS on ICAM-1 expression by SHR, WKY, and SD cerebromicrovascular endothelial cells (EC). Cerebrovascular EC lines isolated from SHR $(\square), W K Y(\square)$ and SD $(\mathbb{\nabla})$ rat brain microvessels were cultured in microtiter plates for three days in the absence or presence of indicated concentrations $(0.1-100$ $\mu \mathrm{g} / \mathrm{ml}$ ) of LPS. EC cultures were examined for ICAM-1 expression by ELISA as described in Materials and Methods. The values presented indicate O.D. \pm S.D. values above those obtained in EC cultures incubated in media alone $(0.241 \pm 0.014,0.277 \pm$ 0.017 and $0.187 \pm 0.016$ for SHR, WKY, and SD EC, respectively); *significant difference $(P<0.01$; Student's $t$ test) compared to SHR EC cultures. 


\section{Discussion}

The experiments here demonstrate the expression of the adhesion molecule, ICAM-1, on cultures of EC isolated from SHR, WKY, and SD rat brain microvessels. Although ICAM-1 expression by brain EC has been previously reported, this is the first demonstration in rats $(10,11,26-28)$. This implicates rat cerebromicrovascular EC in interactions with cells expressing CD1 1a or CD11b integrins (i.e., lymphocytes, monocytes, and neutrophils) $(1,13-16)$. Interactions between these various cell types and EC and the possible role of ICAM-1 in these adhesive interactions has been described in various disorders including rheumatoid arthritis, autoimmune murine lupus nephritis, experimental autoimmune encephalomyelitis (EAE), multiple sclerosis (MS), and atherosclerosis, and in ischemia-reperfusion injury models $(5,7-12,26-28)$.

The possible role of TNF $\alpha$, IL-1 $\beta$, and IFN $\gamma$ in pathogenic processes has been suggested in a number of disorders including EAE, MS, rheumatoid arthritis, and atherosclerosis $(11,29-32)$. The capacity of these cytokines to enhance ICAM-1 expression is well documented $(2,16,18)$ and implicates ICAM-1 or possibly other adhesion molecules in the pathogenesis of these disorders. This may account for why cytokines are often referred to as "double-edged swords" and why mechanisms to inhibit synthesis or actions of cytokines are being explored in order to achieve therapeutic benefits (see review 33).

In all experiments using suboptimal concentrations of cytokines or LPS, the level of up-regulated ICAM-1 expression was greater for SHR EC than for WKY or SD EC. Although the quantitative responses of various EC cultures differed, it was consistently observed that the relative degree of ICAM-1 upregulation by $\mathrm{TNF} \alpha>\mathrm{IL}-1 \beta>\mathrm{IFN} \gamma$. The maximum levels of ICAM-1 expression by SHR, WKY, and SD EC cultures were similar in cultures incubated with optimal concentrations of these cytokines. These data indicate possible differences in the sensitivity of these various EC cultures to cytokine treatment. The observation that optimal, as well as suboptimal, concentrations of LPS up-regulated ICAM-1 expression on SHR EC to a greater extent than on either WKY or SD EC indicates a possible difference in the mechanism(s) by which these EC respond to LPS as opposed to cytokines. Such differences may be influenced by the number of LPS binding sites and possibly the affinity of these sites. The functional significance of the observations described here is indicated by preliminary experiments showing similar findings regarding enhanced adhesion of monocytes. The increased adhesion of monocytes to either LPS- or TNF $\alpha$ - and IL- $1 \beta$-treated SHR EC was significantly higher than in WKY EC ( $P$ $<0.001)$.
The comparison of expression of adhesion receptors by cerebromicrovascular EC from normotensive (WKY and SD) and spontaneously hypertensive (SHR) rats provides a unique opportunity to examine the contribution of hypertension to immune responsiveness, or vice versa. There is already considerable evidence for a relationship between these parameters (34). Although differences between the in vitro responsiveness of the SHR EC and WKY or SD EC cultures were observed only at suboptimal concentrations of cytokines, more differences could exist in vivo, where endothelium is subjected to blood flow/shear stress, factors related to hypertension and various agents including cytokines which may be rapidly washed away. Such in vivo differences are indicated by observations that LPS induces greater increases in plasma levels of TNF $\alpha$ in SHR than in WKY rats (35). Additional studies regarding the influence of hypertension, as well as other potential risk factors for stroke, on expression of adhesion molecules by endothelium may provide insight toward defining a mechanism by which these factors contribute to the initiation of vascular injury.

1. Cavender DE. Interactions between endothelial cells and the cells of the immune system. Int Rev Exp Pathol 32:57-94, 1991.

2. Pober JS. Cytokine-mediated activation of vascular endothelium. Physiology and pathology. Am J Pathol 133:426-433, 1988.

3. Carlos TM, Harlan JM. Membrane proteins involved in phagocyte adherence to endothelium. Immunol Rev 114:5-28, 1990.

4. Harlan JM. Leukocyte-endothelial interactions. Blood 65:513$525,1985$.

5. Wuthrich RP, Jevnikar AM, Takei F, Glimcher LH, Kelly VE. Intercellular adhesion molecule-1 (ICAM-1) expression is upregulated in autoimmune murine lupus nephritis. Am J Pathol 136:441-450, 1990.

6. Lopex-Virella MF, Virella G. Immunological and microbiological factors in the pathogenesis of atherosclerosis. Clin Immunol Immunopathol 37:377-386, 1985.

7. Vedder NB, Winn RK, Rice CL, Chi EY, Arfors KE, Harlan JM. Inhibition of leukocyte adherence by anti-CD18 monoclonal antibody attenuates reperfusion injury in the rabbit ear. Proc Natl Acad Sci USA 87:2643-2646, 1990.

8. Mileski WJ, Winn RK, Vedder NB, Pohlman TH, Harlan JM, Rice CL. Inhibition of CD18-dependent neutrophil adherence reduces organ injury after hemorrhagic shock in primates. Surgery 108:206-212, 1990.

9. Clark WM, Madden KP, Rothlein R, Zivin JA. Reduction of central nervous system ischemic injury in rabbits using leukocyte adhesion antibody treatment. Stroke 22:877-883, 1991.

10. Cannella B, Cross AH, Raine CS. Upregulation and coexpression of adhesion molecules correlate with relapsing autoimmune demyelination in the central nervous system. J Exp Med 172:1521-1524, 1990.

11. Sobel RA, Mitchell ME, Fondren G. Intercellular adhesion molecule-1 (ICAM-1) in cellular immune reactions in the human central nervous system. Am J Pathol 136:1309-1316, 1990.

12. Poston RN, Haskard DO, Coucher JR, Gall NP, Johnson-Tidey RR. Expression of intercellular adhesion molecule-1 in atherosclerotic plaques. Am J Pathol 140:665-673, 1992.

13. Munro JM, Pober JS, Cotran RS. Tumor necrosis factor and gamma interferon induce distinct patterns of endothelial activa- 
tion and associated leukocyte accumulation in skin of Papio anubis. Am J Pathol 135:121-133, 1989.

14. Makgoba MW, Sanders ME, Luce GEG, Dustin ML, Springer TA, Clark EA, Mannoni P, Shaw S. ICAM-1 a ligand for LFA1-dependent adhesion of $B, T$, and myeloid cells. Nature 331:86-88, 1988.

15. Dustin ML, Rothlein R, Bhan AK, Dinarello CA, Springer TA. Induction by IL- 1 and interferon- $\gamma$ : Tissue distribution, biochemistry, and function of a natural adherence molecule (ICAM-1). J Immunol 137:245-254, 1986.

16. Dustin ML, Springer TA. Lymphocyte function-associated antigen-1 (LFA-1) interaction with intercellular adhesion molecule-1 (ICAM-1) is one of at least three mechanisms for lymphocyte adhesion to cultured endothelial cells. J Cell Biol 107:321-331, 1988.

17. Patarroyo M, Makgoba MW. Leukocyte adhesion to cells. Molecular basis, physiological relevance, and abnormalities. Scand J Immunol 30:129-164, 1989.

18. Pober JS, Gimbrone MA, Lapierre LA, Mendrick DL, Fiers W, Rothlein R, Springer TA. Overlapping patterns of activation of human endothelial cells by interleukin 1, tumor necrosis factor, and immune interferon. J Immunol 137:1893-1896, 1986.

19. Colic M, Drabek D. Expression and function of intercellular adhesion molecule 1 (ICAM-1) on rat thymic macrophages in culture. Immunol Lett 28:251-258, 1991.

20. Renonken R, Mennander A, Mattila P, Ustinov J. Signal transduction during in vitro lymphocyte homing. Human Immunol 28:134-140, 1990.

21. May MJ, Ager A. ICAM-1-independent lymphocyte transmigration across high endothelium: Differential up-regulation by interferon $\gamma$, tumor necrosis factor- $\alpha$ and interleukin $1 \beta$. Eur $J$ Immunol 22:219-226, 1992.

22. Tamatani T, Miyasaka M. Identification of monoclonal antibodies reactive with the rat homolog of ICAM-1 and evidence for a differential involvement of ICAM-1 in the adherence of resting versus activated lymphocytes to high endothelial cells. Int Immunol 2:165-171, 1990.

23. Doron DA, Jacobowitz DM, Heldman E, Feuerstein G, Pollard HB, Hallenbeck JM. Extracellular matrix permits the expression of von Willebrand's factor, di-I-acetylated low-density lipoprotein uptake and prostacyclin secretion in cultures of endothelial cells from rat brain microvessels. In Vitro Cell Dev Biol 27A:689-697, 1991.
24. McCarron RM, Spatz M, Kempski O, Hogan RN, Muehl L, McFarlin DE. Interaction between myelin basic proteinsensitized $\mathrm{T}$ lymphocytes and murine cerebral vascular endothelial cells. J Immunol 137:3428-3435, 1986.

25. Tanaka M, McCarron RM. The inhibitory effect of tumor necrosis factor and interleukin-1 on Ia induction by interferon- $\gamma$ on endothelial cells from murine central nervous system microvessels. J Neuroimmunol 27:209-215, 1990.

26. Fabry Z, Waldschmidt MM, Hendrickson D, Keiner J, LoveHoman L, Takei F, Hart MN. Adhesion molecules on murine brain microvascular endothelial cells: Expression and regulation of ICAM-1 and Lgp 55. J Neuroimmunol 36:1-11, 1992.

27. Wilcox CE, Ward AMV, Evans A, Baker D, Rothlein R, Turk JL. Endothelial cell expression of the intercellular adhesion molecule-1 (ICAM-1) in the central nervous system of guinea pigs during acute and chronic relapsing experimental allergic encephalomyelitis. J Neuroimmunol 30:43-51, 1990.

28. O'Neill JK, Butter C, Baker D, Gschmeissner SE, Kraal C, Butcher EC, Turk JL. Expression of vascular addressins and ICAM-1 by endothelial cells in the spinal cord during chronic relapsing experimental allergic encephalomyelitis in the Biozzi AB/H Mouse. Immunol 72:520-525, 1991.

29. Ruddle NH, Bergman CM, McGrath KM, Lingenheld EG, Grunnet ML, Padula SJ, Clark RB. An antibody to lymphotoxin and tumor necrosis factor prevents transfer of experimental allergic encephalomyelitis. J Exp Med 172:1193-1200, 1990.

30. Maimone D, Gregory S, Arnason BGW, Reder AT. Cytokine levels in the cerebrospinal fluid and serum of patients with multiple sclerosis. J Neuroimmunol 32:67-74, 1991.

31. Moyer CF, Sajuthi D, Tulli H, Williams JK. Synthesis of IL-1 alpha and IL-1 beta by arterial cells in atherosclerosis. Am J Pathol 138:951-960, 1991.

32. Pittipher ER, Higgs GA, Henderson B. Interleukin-1 induces leukocyte infiltration and cartilage proteoglycan degradation in the synovial joint. Proc Natl Acad Sci USA 83:8749-8753, 1986.

33. Henderson B, Blake $S$. Therapeutic potential of cytokine manipulation. TiPS 13:145-152, 1992.

34. Dzielak DJ. Immune mechanisms in experimental and essential hypertension. Am J Physiol 260:R459-R467, 1991.

35. Hallenbeck JM, Dutka AJ, Vogel SN, Heldman E, Doran DA, Feuerstein G. Lipopolysaccharide-induced production of tumor necrosis factor activity in rats with and without risk factors for stroke. Brain Res 541:115-120, 1991. 